\title{
Is there decreased activation of deep gray matter structures in multiple sclerosis before cognitive impairment occurs?
}

Martin Berghoff ( $\square$ martin.berghoff@neuro.med.uni )

Justus-Liebig-University of Giessen

Bianca Wagner

Justus-Liebig-University of Giessen

Clara L. Härig

Justus-Liebig-University of Giessen

Bertram Walter

Justus-Liebig-University of Giessen

Jens Sommer

Philipp University of Marburg

Gebhard Sammer

Justus-Liebig-University Giessen

\section{Research Article}

Keywords:

Posted Date: February 18th, 2022

DOI: https://doi.org/10.21203/rs.3.rs-1358102/v1

License: (1) This work is licensed under a Creative Commons Attribution 4.0 International License.

Read Full License 


\title{
Is there decreased activation of deep gray matter structures in multiple sclerosis before cognitive impairment occurs?
}

\author{
Bianca Wagner ${ }^{1}$, Clara L. Härig ${ }^{1}$, Bertram Walter ${ }^{2}$, Jens Sommer ${ }^{3}$, Gebhard \\ Sammer ${ }^{4,5, *}$, Martin Berghoff ${ }^{1, *}$
}

${ }^{1}$ Department of Neurology, Justus-Liebig-University of Giessen, Klinikstrasse 33, 35385 Giessen, Germany

${ }^{2}$ Bender Institute of Neuroimaging, Justus-Liebig-University of Giessen, Otto-BehaghelStrasse 10H, 35394 Giessen, Germany

${ }^{3}$ Department of Psychiatry, University of Marburg, Rudolf-Bultmann-Strasse 8, 35039 Marburg

${ }^{4}$ CognitiveNeuroScience at the Centre of Psychiatry, Justus-Liebig-University of Giessen, Klinikstrasse 36, 35392 Giessen, Germany

${ }^{5}$ Department of Psychology, Justus-Liebig-University of Giessen, Otto-Behaghel-Strasse 10F, 35394 Giessen, Germany

Keywords: Multiple sclerosis, fMRI, cognitive impairment, attention network test

*Corresponding authors:

Prof. Dr. Martin Berghoff

Department of Neurology

Justus-Liebig-University Giessen

Klinikstrasse 33

35385 Giessen

Germany

Email: martin.berghoff@neuro.med.unigiessen.de

Phone: +4964198544306

Fax: +4964198545329
Prof. Dr. Gebhard Sammer

Department of Psychiatry

Justus-Liebig-University Giessen

Klinikstrasse 36

35385 Giessen

Germany

Email: gebhard.sammer@uni-giessen.de

Phone: +4964145835

Fax: +496419945789 


\section{Abstract}

Cognitive impairments related to changes in the deep gray matter (DGM) and other brain regions occur in up to $70 \%$ of patients with multiple sclerosis. Are such brain changes preceded by cognitive decline in patients without clinically evident cognitive impairment? Eighteen participants with relapsing remitting multiple sclerosis (RRMS) and 15 healthy controls took part in this study. Cognitive deficits, depression and fatigue were assessed using the MUSIC test, BDI-II and FSS. FMRI was performed while the participant performed the modified attention network test (ANT). The main analysis concerned the effects of ANT task complexity on the hemodynamic activation of DGMs, including the hippocampus, anterior cingulate cortex (ACC), thalamus, caudate nucleus, pallidum, and putamen. The individual lesion burden was estimated. The group with RRMS showed decreased activation with increasing task complexity in hippocampus, pallidum, and ACC compared to the control group. The thalamus was involved in both group activations but did not differ between groups. Functional changes in the DGM can be detected in RRMS patients before cognitive deficits appear. The affected DGM regions can best be assigned to the Attention Network for Executive Control. This association could serve as a biological indicator of cognitive impairment in MS.

\section{Abstract Word Count: 197 words}

Total Word Count: 2720 Words 


\section{Introduction}

Multiple sclerosis (MS) is a chronic inflammatory and demyelinating disease of the central nervous system. Up to $70 \%$ of patients suffer from cognitive impairment, including deficits in attention, information processing, executive functioning, verbal fluency or long-term memory. ${ }^{1,2}$ Deficits in attention and information-processing are the most common. ${ }^{2,3}$ Cognitive impairment can occur within the first months of the disease ${ }^{4}$, the prevalence of cognitive impairment increases with disease duration, it is more frequent in patients with progressive MS. ${ }^{5}$ Unsurprisingly, cognitive impairment can affect a patient's self-esteem, social functioning, ability to work and quality of life. ${ }^{1,5,6}$ For this reason, it is important to understand how cognitive impairment develop in affected patients in order to be able to predict the underlying signaling pathways earlier and intervene in malfunctions.

Deep grey matter (DGM) pathology has been linked to cognitive impairment in Lewy body dementia $^{7}$, Alzheimer disease ${ }^{8}$ and MS. ${ }^{2}$ Indeed, analyses of autopsy tissue of patients with MS revealed demyelinated lesions and atrophy of various DGM regions. ${ }^{9,10}$ Furthermore, neuronal loss was found in DGM lesions and non-demyelinated DGM regions. ${ }^{10}$ Neuroimaging studies found atrophy of the subcortical DGM in patients with early-stage MS. ${ }^{11}$ The atrophy of DGM nuclei (thalamus, caudate, putamen, pallidum) and the hippocampus correlated with cognitive impairment in patients with RRMS. ${ }^{2}$ A significant correlation between cortical atrophy and poor performance in verbal memory, attention, and verbal fluency was also reported in patients with RRMS and cognitive impairment. ${ }^{12}$ Koenig et al. reported a decrease in hippocampal volume, which correlated with decreases in episodic memory, attention, and processing speed (determined by the magnetization transfer ratio). ${ }^{13}$ Others have shown that subcortical grey matter T2 hypointensity, particularly in the nucleus caudate, pallidum, putamen, and thalamus, is associated with cognitive impairment in patients with MS. ${ }^{14}$ Taken together, these data suggest that degeneration of DGM structures is associated with cognitive impairment in MS.

This is supported by fMRI studies, which show that MS patients without cognitive impairment exhibited more distributed cortical recruitment and increased cerebral activation for cognitive tasks, as well as altered functional connectivity within cognitive networks than in healthy controls. ${ }^{16}$ Another study showed that patients with RRMS had altered activation patterns in tasks that require sustained attention, information processing, and memory: fMRI 
activation was greater in patients with better cognitive function than in those with less cognitive function. When using the Paced Auditory Serial Addition test, functional changes were found in the right complementary motor area, the cingulate cortex, and bilaterally in the prefrontal, temporal and parietal areas. These changes varied with increasing tissue damage. This suggests an adaptive mechanism in response to the underlying neural disorganization or disinhibition. ${ }^{17}$ Others have shown that patients with long-term MS and mild cognitive impairment show increased and additional activation of the frontal cortex and the posterior parietal cortex during neuropsychological tests. This effect decreased with the complexity of the task and was most pronounced for the alertness task. These findings were interpreted as a compensation through the functional integration of frontal and parietal association areas. ${ }^{18}$ The Attention Network Test (ANT) was developed to measure various attention networks, such as alertness, orientation and executive control. ${ }^{19}$ The ANT has since been used in syndromes such as depression ${ }^{20}$ and fatigue ${ }^{21}$, in which patients often have cognitive impairments. In patients with RRMS, the use of the ANT revealed slower reaction times, indicating an impairment of the alerting network. ${ }^{22}$

Despite extensive studies on cognitive impairment in MS, the early changes that underlie the pathology remain unclear. The aim was therefore to investigate functional changes in the DGM structures in RRMS patients without cognitive deficits using the ANT as an activation task. We hypothesized that activation of DGM structures, including the hippocampus, caudate nucleus, anterior cingulate cortex (ACC), thalamus, pallidum, and putamen, decreased with increasing task complexity and slower response time. 


\section{Methods}

\section{Study subjects}

Twenty right-handed participants with RRMS were recruited from the outpatient clinic for multiple sclerosis and neuroimmunology at the Department of Neurology, Justus-LiebigUniversity, Giessen. These patients were diagnosed with RRMS according to the revised McDonald criteria. ${ }^{23}$ A community sample $(n=15)$ was recruited as control group. For both groups, inclusion criteria were right-handedness, age between 18 and 60 years and an EDSS $\leq$ 4.5. ${ }^{24}$ Key exclusion criteria were moderate or marked cognitive dysfunction and moderate or severe depression.

The characteristics of the groups of participants are shown in Tables 1 and 2 . In the patient group, seven participants showed relevant symptoms of fatigue (FSS $\geq 4$ ), two patients had mild depressive symptoms (BDI II score between 13-19 points), two patients showed mild cognitive dysfunction (MUSIC-test score between 16-19 points). One patient performed poorly in the MUSIC-test because of a language barrier. Fourteen patients had low levels of vitamin D3 (25OHD3; normal range 16-74 ng/ml), one patient had a high level of TSH (normal range 0.4-2.5 mU/1). Two patients were excluded from the study because of moderate depression or severe cognitive deficits. In the control group, six participants had decreased levels of vitamin D3, and three controls showed increased levels of TSH.

The study was approved by the ethics committee of Justus-Liebig-University, Giessen $(01 / 14)$. All participants gave their written informed consent. The experimental procedures were carried out in accordance with the Helsinki Declaration.

\section{Study procedures}

All investigations were carried out in the Bender Institute of Neuroimaging, Justus-LiebigUniversity, Giessen, between $4 \mathrm{pm}$ and $7 \mathrm{pm}$. The Multiple Sclerosis Inventory of Cognition (MUSIC) ${ }^{25}$, the Beck Depression Inventory-II (BDI II) ${ }^{26}$, and Fatigue Severity Scale (FSS) ${ }^{27}$ were applied to all study subjects. Complete blood cell count, thyroid hormones (TSH, T3, T4) and vitamin D3 levels were measured in all study subjects (this part of the study will be published elsewhere). The Attention Network Test (ANT) was used to assess cognitive load and attentional performance during functional imaging. The ANT, based on a model of Posner and colleagues ${ }^{19}$, modifications and the course of the task are shown in Figure 1. Study subjects were asked to pay attention and to indicate as quickly as possible the direction of the 
middle arrow using buttons. The task was presented within a block design. The design consisted of three blocks, each block consisted of 96 task trials. The total duration of the task was about 24 minutes depending on how quick the participant was. During fMRI, this task was presented, using Presentation Version 17.2 (NeuroBehavioral Systems, Berkeley, CA, USA). For each participant, reaction times (RT) were averaged over trials for each condition (congruent, incongruent, neutral). RTs were analyzed using a within-subject ANOVA for repeated measures. Between-factor was group (patients, controls), within factors were condition (congruent, incongruent and neutral) and time (three blocks). When appropriate, Greenhouse-Geisser correction was calculated for the within-subjects factors. Least significant differences (LSD), which control for family-wise error, were calculated for posthoc comparisons. ${ }^{28}$

IBM SPSS Version 26 was used computing the statistics. For each trial the accuracy of the response (HIT, MISS) was recorded as an additional behavioral measure.

\section{Image acquisition, functional image and lesion analyses}

Brain images were acquired using a 3T Siemens Prisma scanner (Siemens, Erlangen, Germany) with a standard 64-channel head coil. The imaging protocol consisted of a gradient echo field map sequence (TR 1000ms, TE1 10 ms, TE2 12.46 ms, flip angle 90 deg.; other parameters see EPI below) for $\mathrm{B}_{0}$-correction, $\mathrm{T} 1$-weighted magnetization prepared rapid acquisition gradient echo (MPRAGE; 176 slices, thickness $0.94 \mathrm{~mm}$, sagittal orientation, FOV $240 \mathrm{~mm}$, phase encoding a>>p, TR $1580 \mathrm{~ms}$, TE $2.3 \mathrm{~ms}$ ) for normalization of brains, and T2*-weighted echo-planar imaging (EPI; max. 590 measurements, 40 slices; dist. Factor 25\%, descending, thickness 3mm, phase encoding a >>p, FOV $220 \mathrm{~mm}$, TR $2500 \mathrm{~ms}$, TE 30 ms, flip angle $85 \mathrm{deg}$, accel. mode GRAPPA) for BOLD imaging during the task performance. Image preprocessing and analyses were performed using the Functional Magnetic Resonance Imaging of the Brain (FMRIB) Software Library (FSL 5.0.9, Feat 6.00) pipelines. Subject level design matrices included the regressors HIT and MISS for each correctly or incorrectly answered task (congruent, incongruent, or neutral), their first derivative, and HIT modulated by congruent, incongruent, or neutral condition. The reaction time was implemented as a modulator to indicate cognitive load. Regressors of no interest were the six head motion parameters and a binary regressor for each outlier volume. For the BOLD images two contrasts were created. The contrast TASK was defined as the difference between HIT (incongruent task), and the mean of HIT (congruent task) and HIT (neutral task). The contrast TIME considered reaction time regardless of the type of task. 
DGM structures (caudate nucleus, anterior cingulate gyrus, thalamus, pallidum, and putamen) and hippocampus were selected as regions of interest (ROI) for analysis. It was defined that voxels with $\mathrm{p}>0.5$ regarding the probabilistic Havard-Oxford brain atlas belonged to that ROI. Areas of activation due to cognitive load or attentional performance were identified by a group-level design modelling the means of the groups. Group comparison was restricted to voxels, showing activation due to mental load to at least one group. Each ROI was considered separately. The same design used to determine areas of activation was used to test the difference between controls and patients (two-sided test).

For the assessment of the individual lesion load from the structural images, the legion segmentation tool (LST) toolbox version 2.0.15 ${ }^{29}$ for SPM $12^{30}$ was used. The lesion growth algorithm (LGA) from the LST was started with an initial threshold of 0.3 (chosen after visual inspection) using T1- and T2-weighted structural images. For two subjects T2-weighted images were not available, so LGA could not be used. The corresponding values are missing in Table 1. More information on image acquisition, functional image analyses, and lesion analyses can be found in the supplementary data.

\section{Results}

\section{Prolonged reaction times in patients}

Response time measurements while performing the ANT were recorded to indicate attention processing. Repeated measurement ANOVA showed that reaction times were generally slower in the MS-group than in the control group (main factor group), in incongruent versus both congruent or neutral conditions (main factor task), and in block 1 (main factor time blocks, post-hoc comparison $\mathrm{p}=0.018$ ). No interaction was found between any of the factors. The reaction time data are shown in Figure 2, the corresponding ANOVA table can be found in Table 3.

\section{Decreased activation in DGM structures in patients}

To investigate effects of task, the contrast TASK was calculated as HIT(incongruent task) $($ HIT (congruent task) + HIT(neutral task $)) / 2$. The contrast TIME considered the reaction time regressor regardless of the task type. In the patient's group, activation by TASK occurred in the thalamus (pFWE $<0.05$ ). In the control group, regional DGM activation by TASK was also found in the thalamus but also in 
the ACC, the caudate nucleus, the hippocampus, the pallidum (all pFWE < 0.05) (Table 4). Contrasting both groups revealed that the activation by TASK in the ACC, hippocampus, and pallidum was lower in patients compared with controls, but not in the thalamus (Table 5). For the contrast TIME, regional DGM activation related to the reaction speed regardless of the type of task was found in the ACC $(\mathrm{pFWE}=0.017$ at $\mathrm{x} / \mathrm{y} / \mathrm{z}=6 / 20 / 32$, cluster size of 31 voxels). However, no effects with group above the threshold were found.

\section{Lesion load in the brain}

To determine the damage caused by inflammation and degeneration in MS, we calculated the lesion load (size and according number of lesions) and extend of white matter hyperintensities (corrected for estimated total intracranial volume) (Table 1). We found a strong correlation between the lesion size and extend of white matter hyperintensities $(\mathrm{p}<0.001)$, which points to a robust lesion estimation by the two different algorithms. However, lesion load correlated with the EDSS $(p=0.037)$, but did not correlate with the MUSIC test $(p=0.875)$, FSS $(p=$ $0.756)$, or the BDI II $(\mathrm{p}=0.629)$.

\section{Discussion}

The most common and disabling cognitive deficits in MS patients are impaired attention, processing speed, and executive functions. These deficits can also appear early in the course of the disease ${ }^{3}$. The present study examined the hemodynamic activation of those DGM structures that are associated with cognitive impairments in patients with relapsing-remitting multiple sclerosis (RRMS). The attention network test (ANT) was carried out during the fMRI in order to induce cognitive task load. Effects of task complexity (TASK) and reaction speed (TIME) on hemodynamic activation were analyzed.

The main result showed a lower activation in the ACC, hippocampus and pallidum in the patients with RRMS. With regard to description of attention networks with the ANT by Fan et al. ${ }^{33}$, this activation pattern indicates reduced executive control while maintaining attention in patients without acute clinically evident cognitive deficits. This was due to the finding that activation of the ACC, caudate nucleus, hippocampus, pallidum and thalamus for task complexity was found in the control group, while in the group of patients with RRMS only 
activation of the thalamus above threshold lay. Regardless of task complexity, reaction speed showed an effect on the anterior cingulate gyrus. However, there was no group effect. This indicates that the alertness function was not impaired in patients or controls. The main finding of the behavioral measures of attention was the slower overall reaction times in the patients. The other effects, namely the acceleration of the reaction times in the course of the task and the time required to solve the tasks of the incongruent condition, are well known and can be understood in terms of a successful manipulation test. These behavioral results are consistent with the work of Urbanek et al. which described a prolonged reaction time in mostly cognitively unaffected patients with RRMS using the ANT. ${ }^{22}$ However, contrary to these studies, one study even reported a decline in attention performance over time. ${ }^{6}$

The results of the current study indicate a reduced activation of some DGM structures in RRMS patients without currently detectable impairment of cognition. These DGM structures include areas related to executive control of attention rather than alertness, since thalamic activity was unimpaired in the patients. These results are in good agreement with studies showing the importance of DGM structures for cognition in multiple sclerosis. ${ }^{34}$ It has been suggested that atrophy of the hippocampus and DGM nuclei may be the best predictor of cognitive impairment. ${ }^{2}$ DGM atrophy was also detected in patients with earlystage MS. ${ }^{11}$ A decrease in total thalamic volume and neuronal density was also observed in patients with multiple sclerosis. ${ }^{32}$ However, the current study found no difference in thalamic activation in cognitively unimpaired patients with RRMS, indicating unimpaired, or at least less impaired, attentional network associated with alertness. Further, basal ganglia have been previously associated with cognitive dysfunction in MS. An association between cognitive dysfunction and structural changes in the caudate nucleus, pallidum and putamen has been reported. ${ }^{14}$ In contrast, studies in a similar group of patients reported increased cerebral activation, expanded cortical recruitment, and changes in the functional connectivity of regions associated with cognitive processing. The increased activation together with structural damage was explained by the forced recruitment of cortical networks to compensate for cognitive deficits. ${ }^{16}$

The brain network approach implies that correlates of cognitive processing are not restricted to deep gray matter structures. Patients with RRMS who had no or only mild cognitive impairment showed greater activation and recruitment of the inferior and middle frontal gyrus, the inferior parietal cortex, the middle and superior temporal gyrus, the anterior 
cingulate cortex, and the basal ganglia. The hemodynamic activation of the brain was higher in patients with better preserved cognitive function. Functional changes seemed to increase with greater tissue damage, which likely suggests that adaptive processes were occurring. ${ }^{17}$ A strong positive correlation between the changes in activation and cerebral lesion burden was reported in studies on motor function. Functional changes in cortical and subcortical motor-related areas can limit impairment caused by brain damage in MS. ${ }^{15}$

In summary, a lower hemodynamic brain activation was found in some DGM structures such as the hippocampus, pallidum or the anterior cingulate cortex, in RRMS patients. These functional changes in DGM regions can be detected before cognitive deficits become evident . The affected structures in patients can be assigned to the attention network for executive control. In contrast, the preserved thalamus activation indicates an at least less impaired attention network "alertness". The results of the study suggest an association between cognitive impairment and changes in functional integrity in DGMs, which could serve as a biological indicator of cognitive impairment in MS.

Acknowledgements: The study was supported by Genzyme GmbH, Neu-Isenburg, Germany.

Conflict of interest: The authors declare no competing financial interests. 


\section{References}

1. Chiaravalloti ND and DeLuca J. Cognitive impairment in multiple sclerosis. The Lancet Neurology 2008; 7: 1139-1151.

2. Damjanovic D, Valsasina P, Rocca MA, et al. Hippocampal and Deep Gray Matter Nuclei Atrophy Is Relevant for Explaining Cognitive Impairment in MS: A Multicenter Study. AJNR Am J Neuroradiol 2017; 38: 18-24.

3. Wybrecht D, Reuter F, Pariollaud F, et al. New brain lesions with no impact on physical disability can impact cognition in early multiple sclerosis: A ten-year longitudinal study. PLoS ONE 2017; 12: e0184650.

4. Nourbakhsh B, Nunan-Saah J, Maghzi A-H, et al. Longitudinal associations between MRI and cognitive changes in very early MS. Mult Scler Relat Disord 2016; 5: 47-52.

5. Deluca GC, Yates RL, Beale H, et al. Cognitive impairment in multiple sclerosis: Clinical, radiologic and pathologic insights. Brain Pathol 2015; 25: 79-98.

6. Winkelmann A, Engel C, Apel A, et al. Cognitive impairment in multiple sclerosis. $J$ Neurol 2007; 254 Suppl 2: II35-42.

7. Firbank MJ, O'Brien JT and Taylor JP. Long reaction times are associated with delayed brain activity in lewy body dementia. Hum Brain Mapp 2018; 39: 633-643.

8. Hagemeier J, Woodward MR, Rafique UA, et al. Odor identification deficit in mild cognitive impairment and Alzheimer's disease is associated with hippocampal and deep gray matter atrophy. Psychiatry Res Neuroimaging 2016; 255: 87-93.

9. Haider L, Simeonidou C, Steinberger G, et al. Multiple sclerosis deep grey matter: The relation between demyelination, neurodegeneration, inflammation and iron. $J$ Neurol Neurosurg Psychiatr 2014; 85: 1386-1395.

10.Vercellino $M$, Masera $S$, Lorenzatti $M$, et al. Demyelination, inflammation, and neurodegeneration in multiple sclerosis deep gray matter. $J$ Neuropathol Exp Neurol 2009; 68: 489-502.

11.Bergsland N, Horakova D, Dwyer MG, et al. Subcortical and cortical gray matter atrophy in a large sample of patients with clinically isolated syndrome and early relapsing-remitting multiple sclerosis. AJNR Am J Neuroradiol 2012; 33: 15731578.

12.Amato MP, Bartolozzi ML, Zipoli V, et al. Neocortical volume decrease in relapsingremitting MS patients with mild cognitive impairment. Neurology 2004; 63: 89-93. 
13.Koenig KA, Sakaie KE, Lowe MJ, et al. Hippocampal volume is related to cognitive decline and fornicial diffusion measures in multiple sclerosis. Magn Reson Imaging 2014; 32: 354-358.

14.Brass SD, Benedict RHB, Weinstock-Guttman B, et al. Cognitive impairment is associated with subcortical magnetic resonance imaging grey matter T2 hypointensity in multiple sclerosis. Mult Scler 2006; 12: 437-444.

15. Mainero C, Pantano P, Caramia F, et al. Brain reorganization during attention and memory tasks in multiple sclerosis: Insights from functional MRI studies. $J$ Neurol Sci 2006; 245: 93-98.

16.Rocca MA, Amato MP, Stefano N de, et al. Clinical and imaging assessment of cognitive dysfunction in multiple sclerosis. The Lancet Neurology 2015; 14: 302317.

17. Mainero $\mathrm{C}$, Caramia F, Pozzilli $\mathrm{C}$, et al. $\mathrm{fMRI}$ evidence of brain reorganization during attention and memory tasks in multiple sclerosis. Neuroimage 2004; 21: 858-867.

18.Penner I-K, Rausch M, Kappos L, et al. Analysis of impairment related functional architecture in MS patients during performance of different attention tasks. J Neurol 2003; 250: 461-472.

19.Fan J, McCandliss BD, Sommer T, et al. Testing the efficiency and independence of attentional networks. J Cogn Neurosci 2002; 14: 340-347.

20.Sommerfeldt SL, Cullen KR, Han G, et al. Executive Attention Impairment in Adolescents With Major Depressive Disorder. J Clin Child Adolesc Psychol 2016; 45: 69-83.

21. Hughes AM, Hirsch CR, Nikolaus S, et al. Cross-Cultural Study of Information Processing Biases in Chronic Fatigue Syndrome: Comparison of Dutch and UK Chronic Fatigue Patients. Int J Behav Med 2018; 25: 49-54.

22.Urbanek C, Weinges-Evers N, Bellmann-Strobl J, et al. Attention Network Test reveals alerting network dysfunction in multiple sclerosis. Mult Scler 2010; 16: 9399.

23.Polman $\mathrm{CH}$, Reingold SC, Banwell B, et al. Diagnostic criteria for multiple sclerosis: 2010 revisions to the McDonald criteria. Ann Neurol 2011; 69: 292-302.

24.Kurtzke JF. Rating neurologic impairment in multiple sclerosis: an expanded disability status scale (EDSS). Neurology 1983; 33: 1444-1452. 
25.Calabrese P, Kalbe E and Kessler J. Ein neuropsychologisches Screening zur Erfassung kognitiver Störungen bei MS-Patienten - Das Multiple Sklerose Inventarium Cognition (MUSIC). psychoneuro 2004; 30: 384-388.

26. Hautzinger $M$, Keller $F$, Kühner $C$, et al. Beck Depressions-Inventar: BDI II.Revision. Revision. Frankfurt am Main: Harcourt Test Services, (2006).

27.Krupp LB. The Fatigue Severity Scale. Arch Neurol 1989; 46: 1121.

28.Shaffer JP. Modified sequentially rejective multiple test procedures. Journal of the American Statistical Association 1986: 826-831.

29.Schmidt P, Gaser C, Arsic M, et al. An automated tool for detection of FLAIRhyperintense white-matter lesions in Multiple Sclerosis. Neuroimage 2012; 59: 3774-3783.

30.Friston KJ. Statistical parametric mapping: The analysis of funtional brain images. 1st ed. Amsterdam, Boston: Elsevier/Academic Press, 2007.

31. Kujala P., Portin R., Revonsuo A., Ruutiainen J.: Attention related performance in two cognitively different subgroups of patients with multiple sclerosis. Journal of neurology, neurosurgery, and psychiatry 59, 77-82 (1995).

32.Cifelli $A$, Arridge $M$, Jezzard $P$, et al. Thalamic neurodegeneration in multiple sclerosis. Ann Neurol 2002; 52: 650-653.

33. Fan J, McCandliss BD, Fossella J, Flombaum JI, Posner MI. The activation of attentional networks. Neuroimage. 2005 Jun;26(2):471-9. doi: 10.1016/j.neuroimage.2005.02.004. Epub 2005 Mar 19. PMID: 15907304.

34. Pinter D, Khalil M, Pichler A, Langkammer C, Ropele S, Marschik PB, Fuchs S, Fazekas F, Enzinger $\mathrm{C}$. Predictive value of different conventional and nonconventional MRI-parameters for specific domains of cognitive function in multiple sclerosis. Neurolmage: Clinical, Volume 7, 2015, 715-720, doi:

10.1016/j.nicl.2015.02.023. 


\section{Statements and Declarations}

\section{Declarations: Author Contributions/Conflict of Interests/Additional Information}

\subsection{Ethics approval and consent to participate}

Each participant or its legal authorized representative was fully informed of this study and gave their written consent to participate. This study was approved by the ethics committee from the Faculty of Medicine of the Justus Liebig University Giessen (file number 01/14) and carried out in accordance with the Helsinki Declaration and the ethical standards of the APA.

\subsection{Consent for publication}

Not applicable

\subsection{Availability of data and materials}

The data sets generated and / or analyzed in the course of the current study are not publicly accessible due to the applicable data protection law of the State of Hesse, but are available on justified request from the corresponding author.

\subsection{Author Contributions}

Bianca Wagner

- Affiliation: Dr. med., Department of Neurology, Justus-Liebig-University of Giessen, Klinikstrasse 33, 35385 Giessen, Germany

- e-mail: bianca.wagner@chiru.med.uni-giessen.de

- Manuscript Contribution: supervised the data collection and carried out the analysis of the questionnaire data, drafted the manuscript, worked on all parts of the manuscript, including proofreading.

Clara L. Härig

- Affiliation: Dr. med, Department of Neurology, Justus-Liebig-University of Giessen, Klinikstrasse 33, 35385 Giessen, Germany

- e-mail: c.haerig@st-vincenz.de

- Manuscript Contribution: supervised the data collection and carried out the analysis of the questionnaire data, drafted the manuscript, worked on all parts of the manuscript, including proofreading.

Bertram Walter

- Affiliation: Dr. phil., Bender Institute of Neuroimaging, Justus-Liebig-University of Giessen, OttoBehaghel-Strasse 10H, 35394 Giessen, Germany

- e-mail: Bertram.Walter@psychol.uni-giessen.de

- Manuscript Contribution: supervising experimental procedures, fMRI data preprocessing and analysis, manuscript proofreading. 
- Dr. Dipl.-Phys., Core Facility Brainimaging, Philipps University Marburg, Germany, Department of Psychiatry, Philipps University Marburg, Rudolf-Bultmann-Strasse 8, 35039 Marburg

- e-mail: sommerj@med.uni-marburg.de

- Manuscript Contribution: analysis of MR-based lesion load, manuscript proofreading.

Gebhard Sammer

- Information: apl. Professor, Dr. phil. habil., Faculty of Medicine - Psychiatry, and Faculty of Psychology and Sports Sciences - Psychology, Justus Liebig University Giessen (JLU), Klinikstrasse 36, 35385 Giessen, Germany

- e-mail: gebhard.sammer@uni-giessen.de

- Manuscript Contribution: GS together with MB developed the idea and details for the study, was involved in setting up the experimental procedure, worked on all parts of the manuscript and on proofreading. Together with MB this author is responsible for everything to do with the submission process on behalf of all authors of the manuscript.

\section{Martin Berghoff}

- Affiliation: apl. Professor, Dr. med. habil., Department of Neurology, Justus-Liebig-University of Giessen, Klinikstrasse 33, 35385 Giessen, Germany

- e-mail: martin.berghoff@neuro.med.uni-giessen.de

- Manuscript Contribution: together with GS developed the idea and details for the study, was involved in setting up the experimental procedure, worked on all parts of the manuscript and on proofreading. Together with GS this author is responsible for everything to do with the submission process on behalf of all authors of the manuscript.

\subsection{Competing Interests}

Non-financial competing interests: The authors declare that they have no competing interests.

Funding: The study was supported by Genzyme GmbH, Neu-Isenburg, Germany. The supporter had no influence on the design, data collection, analysis, interpretation of the results, or any other aspect of the study.

Employment: The authors of this study were not hired by any organization that may gain or lose financially through this publication.

Personal financial interests: MB received grants or contracts from Novartis; consulting fees from Sanofi-Aventis Dtl. GmbH, Novartis Pharma GmbH, Janssen-Cilag GmbH, Roche Pharma AG, Merck Serono $\mathrm{GmbH}$, Biogen $\mathrm{GmbH}$; payment or honoraria for lectures, presentations, speakers bureaus, manuscript writing or educational events from Sanofi-Aventis Dtl. GmbH, Novartis Pharma GmbH, Roche Pharma AG, Alexion Pharma GmbH, Merck Serono $\mathrm{GmbH}$; support for attending meetings and/or travel from Celgene $\mathrm{GmbH}$, Biogen $\mathrm{GmbH}$, Novartis Pharma GmbH.

The other authors have not received any compensation (monetary, shares, etc.) from any company, consulting fees, or any other form of remuneration from any organization. 
Table 1 Patient characteristics

\begin{tabular}{|c|c|c|c|c|c|c|c|c|c|c|c|}
\hline Patient & $\begin{array}{c}\text { Sex } \\
(\mathbf{M} / \mathbf{F})\end{array}$ & $\begin{array}{c}\text { Age } \\
\text { (years) }\end{array}$ & $\begin{array}{c}\text { Years since } \\
\text { diagnosis }\end{array}$ & EDSS & FSS & BDI II & MUSIC & $\begin{array}{l}\text { LGA } \\
\text { TLV }\end{array}$ & $\begin{array}{c}\text { LGA } \\
\text { n }\end{array}$ & $\begin{array}{c}\text { WMH } \\
\text { Volume }\end{array}$ & Disease modifying therapy \\
\hline 1 & M & 23 & 2 & 0.0 & 4.8 & 9 & 16 & na & na & 1250.9 & Teriflunomide $14 \mathrm{mg}$ \\
\hline 2 & M & 33 & 5 & 0.0 & 2.1 & 2 & 25 & 1.87 & 13 & 2185.2 & IFN $\beta-1 \mathrm{a} 22 \mu \mathrm{g}$ \\
\hline 3 & $\mathrm{~F}$ & 54 & 24 & 1.5 & 1.1 & 0 & 27 & 5.03 & 20 & 3065.0 & Teriflunomide $14 \mathrm{mg}$ \\
\hline 4 & M & 37 & 6 & 0.0 & 1.8 & 0 & 28 & 0.22 & 6 & 699.7 & IFN $\beta-1 \mathrm{a} 22 \mu \mathrm{g}$ \\
\hline 5 & $\mathrm{~F}$ & 51 & 8 & 1.0 & 1.9 & 2 & 23 & na & na & 1098.1 & Teriflunomide $14 \mathrm{mg}$ \\
\hline 6 & $\mathrm{~F}$ & 33 & 2 & 1.5 & 5.9 & 11 & 29 & 0.20 & 5 & 566.0 & IFN $\beta-1 \mathrm{a} 44 \mu \mathrm{g}$ \\
\hline 7 & $\mathrm{~F}$ & 55 & 25 & 3.0 & 5.0 & 19 & 25 & 1.16 & 19 & 1192.7 & IFN $\beta-1 \mathrm{a} 44 \mu \mathrm{g}$ \\
\hline 8 & $\mathrm{~F}$ & 60 & 5 & 1.0 & 2.9 & 5 & 17 & 0.08 & 2 & 815.9 & IFN $\beta-1 \mathrm{a} 22 \mu \mathrm{g}$ \\
\hline 9 & $\mathrm{~F}$ & 46 & 3 & 2.0 & 2.7 & 4 & 27 & 7.95 & 26 & 4741.1 & IFN $\beta-1 \mathrm{a} 22 \mu \mathrm{g}$ \\
\hline 10 & $\mathrm{~F}$ & 36 & 3 & 2.5 & 6.4 & 17 & 25 & 0.26 & 5 & 543.4 & IFN $\beta-1 \mathrm{a} 22 \mu \mathrm{g}$ \\
\hline 11 & $\mathrm{~F}$ & 54 & 11 & 2.5 & 5.2 & 0 & 26 & 2.82 & 24 & 1607.8 & IFN $\beta-1 \mathrm{~b} 250 \mu \mathrm{g}$ \\
\hline 12 & $\mathrm{~F}$ & 52 & 9 & 1.0 & 5.2 & 7 & 28 & 0.68 & 12 & 1202.2 & IFN $\beta-1 \mathrm{~b} 250 \mu \mathrm{g}$ \\
\hline 13 & $\mathrm{M}$ & 49 & 10 & 4.5 & 4.6 & 10 & 23 & 5.31 & 24 & 4433.4 & IFN $\beta-1 \mathrm{~b} 250 \mu \mathrm{g}$ \\
\hline 14 & $\mathrm{~F}$ & 43 & 6 & 1.0 & 1.4 & 7 & $14^{1}$ & 1.02 & 17 & 1276.6 & IFN $\beta-1 \mathrm{a} 22 \mu \mathrm{g}$ \\
\hline 15 & $\mathrm{M}$ & 32 & 6 & 1.0 & 1.7 & 11 & 27 & 0.98 & 11 & 677.3 & IFN $\beta-1 \mathrm{a} 44 \mu \mathrm{g}$ \\
\hline 16 & $\mathrm{~F}$ & 26 & 3 & 0.0 & 2.1 & 1 & 28 & 0.09 & 3 & 474.5 & IFN $\beta-1 \mathrm{a} 22 \mu \mathrm{g}$ \\
\hline 17 & $\mathrm{~F}$ & 38 & 10 & 0.0 & 3.7 & 9 & 29 & 0.70 & 12 & 1387.1 & IFN $\beta-1 \mathrm{a} 44 \mu \mathrm{g}$ \\
\hline 18 & $\mathrm{~F}$ & 33 & 10 & 1.0 & 1.9 & 0 & 24 & 0.15 & 4 & 666.4 & IFN $\beta-1 \mathrm{a} 22 \mu \mathrm{g}$ \\
\hline $\begin{array}{c}\text { Mean } \\
\pm \\
\text { SD }\end{array}$ & & $\begin{array}{c}42.0 \\
\pm \\
11.0\end{array}$ & $\begin{array}{c}8.1 \\
\pm \\
6.6\end{array}$ & $\begin{array}{c}1.3 \\
\pm \\
1.2\end{array}$ & $\begin{array}{c}3.4 \\
\pm \\
1.7\end{array}$ & $\begin{array}{c}6.3 \\
\pm \\
5.8\end{array}$ & $\begin{array}{c}24.5 \\
\pm \\
4.5\end{array}$ & & & & \\
\hline
\end{tabular}

${ }^{1}$ language barrier

na: not available, TLV: total lesion volume 
Table 2 Healthy control characteristics

\begin{tabular}{|c|c|c|c|c|c|}
\hline Subject & $\begin{array}{l}\text { Sex } \\
(M / F)\end{array}$ & $\begin{array}{c}\text { Age } \\
\text { (years) }\end{array}$ & FSS & BDI II & MUSIC \\
\hline 1 & $\mathrm{M}$ & 22 & 2.3 & 2 & 30 \\
\hline 2 & F & 23 & 3.7 & 3 & 30 \\
\hline 3 & $\mathrm{M}$ & 29 & 3.1 & 3 & 23 \\
\hline 4 & $\mathrm{~F}$ & 39 & 2.3 & 4 & 24 \\
\hline 5 & $\mathrm{~F}$ & 24 & 1.1 & 1 & 30 \\
\hline 6 & $\mathrm{~F}$ & 30 & 1.9 & 1 & 30 \\
\hline 7 & $\mathrm{M}$ & 24 & 1.6 & 0 & 30 \\
\hline 8 & $\mathrm{~F}$ & 33 & 2.0 & 1 & 30 \\
\hline 9 & $\mathrm{M}$ & 35 & 2.1 & 0 & 26 \\
\hline 10 & $\mathrm{M}$ & 43 & 2.7 & 7 & 29 \\
\hline 11 & $\mathrm{~F}$ & 31 & 2.8 & 1 & 30 \\
\hline 12 & $\mathrm{M}$ & 29 & 1.8 & 6 & 30 \\
\hline 13 & $\mathrm{~F}$ & 29 & 1.3 & 2 & 28 \\
\hline 14 & $\mathrm{~F}$ & 29 & 3.4 & 5 & 27 \\
\hline 15 & $\mathrm{M}$ & 51 & 2.2 & 1 & 25 \\
\hline Mean & & 31.4 & 2.3 & 2.5 & 28.1 \\
\hline$\stackrel{ \pm}{\mathrm{SD}}$ & & \pm & \pm & \pm & \pm \\
\hline SW & & 8.0 & 0.7 & 2.2 & 2.5 \\
\hline
\end{tabular}


Table 3 Reaction time analysis with factors group, task, and time block: F, df (Greenhouse-Geisser corrected for within-subject effects), $p$ and partial $\mathrm{Eta}^{2}$

\begin{tabular}{lllll} 
Effects & $\mathbf{F}$ & $\mathbf{d f}$ & $\boldsymbol{p}$ & $\mathbf{p a r t i a l ~}_{\mathbf{E t a}}{ }^{2}$ \\
\hline Group & 12.20 & $1 ; 31$ & 0.001 & 0.282 \\
\hline Task & 211.63 & $1.82 ; 56.29$ & $<0.001$ & 0.872 \\
\hline Time block & 5.00 & $1.47 ; 45.64$ & 0.018 & 0.139 \\
\hline Interactions & & & & \\
\hline Group * Task & 0.76 & $1.82 ; 56.29$ & 0.460 & 0.024 \\
\hline Group * Time block & 1.29 & $1.47 ; 45.64$ & 0.277 & 0.040 \\
\hline Task * Time block & 2.21 & $3.19 ; 98.76$ & 0.087 & 0.067 \\
\hline Group * Task * Time block & 0.76 & $3.19 ; 98.76$ & 0.526 & 0.024
\end{tabular}


Table 4 Activation by TASK in healthy controls (HC) and patients with RRMS (MS): Results of permutation tests using threshold-free cluster enhancement. Clusters containing only voxels with $\mathrm{p}_{\mathrm{FWE}}<0.05$ and their maximum with MNI coordinates are shown.

\begin{tabular}{|c|c|c|c|c|c|c|}
\hline Group & Region & $\begin{array}{l}\text { Cluster size } \\
\text { (voxels) }\end{array}$ & pFWE & $\mathbf{x}$ & $y$ & $\mathbf{z}$ \\
\hline \multicolumn{7}{|l|}{$\mathrm{HC}$} \\
\hline \multirow{2}{*}{\multicolumn{2}{|c|}{ ACC }} & 179 & 0.001 & 6 & 28 & 24 \\
\hline & & 201 & 0.008 & 2 & 12 & 32 \\
\hline \multirow{2}{*}{\multicolumn{2}{|c|}{ Hippocampus }} & 94 & 0.005 & 34 & -18 & -16 \\
\hline & & 1 & 0.048 & -26 & -28 & -10 \\
\hline & Pallidum & 8 & 0.024 & 16 & -4 & -6 \\
\hline & Saudate nucleus & 20 & 0.009 & 12 & 6 & 12 \\
\hline \multirow{2}{*}{\multicolumn{2}{|c|}{ Thalamus }} & 255 & 0.003 & -10 & -8 & 10 \\
\hline & & 209 & 0.005 & 12 & -12 & 6 \\
\hline \multicolumn{7}{|l|}{ MS } \\
\hline \multirow{2}{*}{\multicolumn{2}{|c|}{ Thalamus }} & 28 & 0.028 & 14 & -10 & 2 \\
\hline & & 1 & 0.048 & 2 & -8 & 2 \\
\hline
\end{tabular}


Table 5 Group differences in activation by TASK in healthy controls (HC) and patients with RRMS (MS). Results of permutation tests using threshold-free cluster enhancement. Clusters containing only voxels with $\mathrm{p}_{\mathrm{FWE}}<0.05$ and their maximum with MNI coordinates are shown. All tests indicate higher activation in $\mathrm{HC}$ than in MS.

\begin{tabular}{rccccc} 
Region & $\begin{array}{c}\text { Cluster size } \\
\text { (voxels) }\end{array}$ & p pWE & x & y & z \\
\hline ACC & 2 & 0.049 & 6 & 28 & 22 \\
Hippocampus & 55 & 0.002 & 32 & -18 & -16 \\
& 8 & 0.022 & 34 & -32 & -8 \\
Pallidum & 8 & 0.003 & 16 & -4 & -6
\end{tabular}


Figure 1

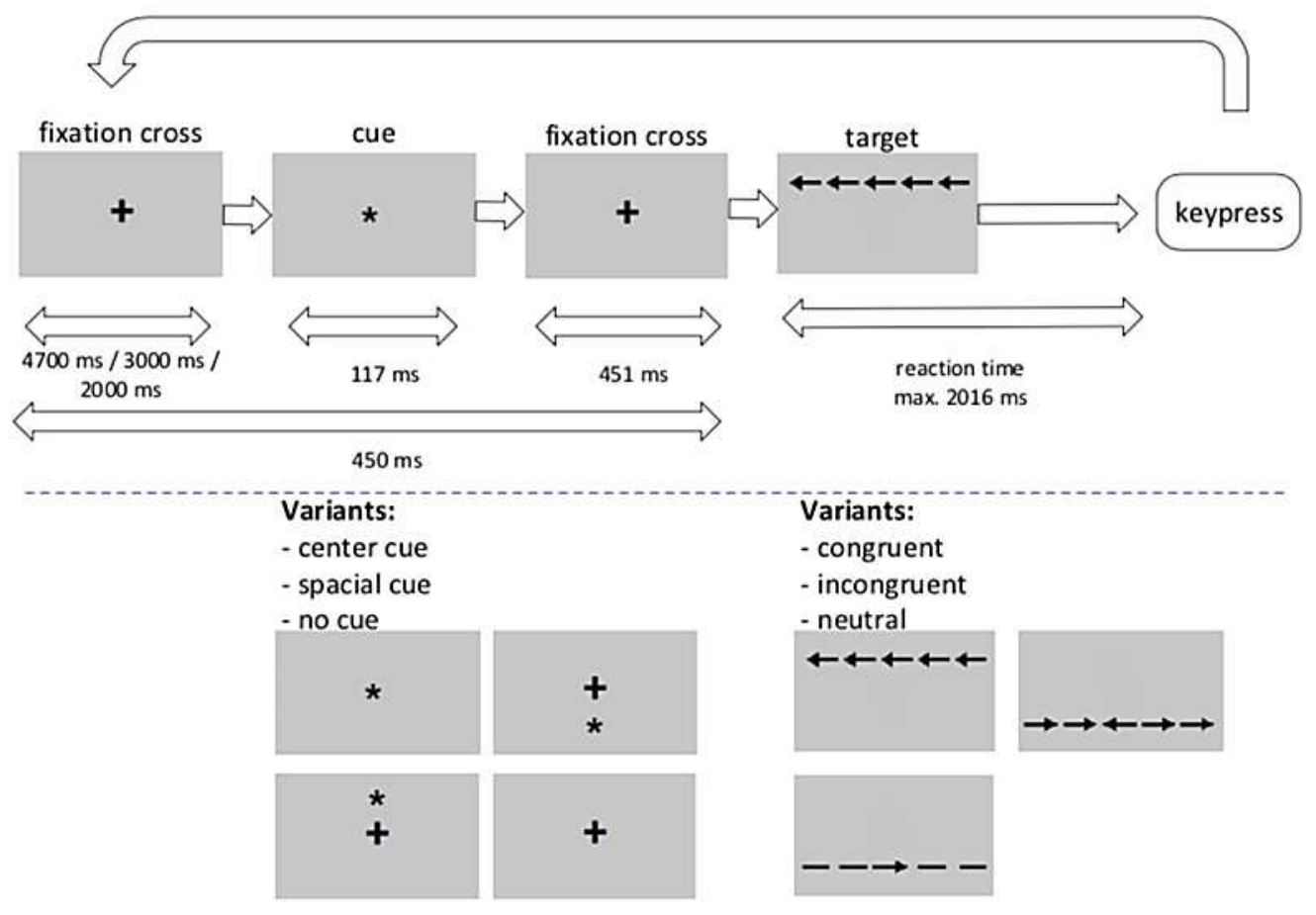

Figure 1 Modified ANT based on the version developed by Posner et al. ${ }^{19}$ Upper panel: Trial structure; lower panel: Task variants

MS

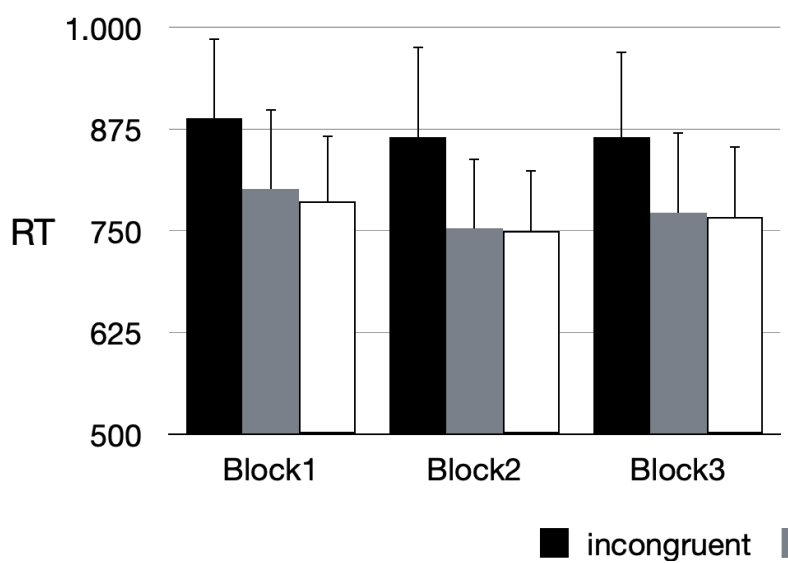

1.000

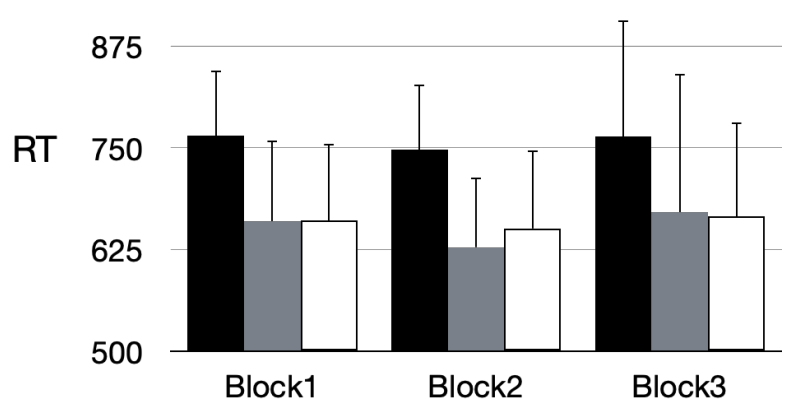

Figure 2 Reaction times for tasks, conditions, and time blocks (Means + SD) 


\section{Supplementary Files}

This is a list of supplementary files associated with this preprint. Click to download.

- WagneretalSupplemetaryMaterial.pdf 